\title{
Remarks to the Standard Theory of Neutrino Oscillations. Corrected Theory of Neutrino Oscillations
}

\author{
Beshtoev Kh. M. \\ Joint Institute for Nuclear Research, Joliot Curie 6, 141980 Dubna, \\ Moscow region, Russia
}

\begin{abstract}
In the Standard theory of neutrino oscillations it is supposed that physical observed neutrino states $\nu_{e}, \nu_{\mu}, \nu_{\tau}$ have no definite masses and that neutrinos are initially created as mixture of $\nu_{1}, \nu_{2}, \nu_{3}$ neutrino states and that neutrino oscillations are the real ones even when neutrino masses are different. It is shown that these suppositions lead to violation of the law of energy and momentum conservation and then the neutrino states are unstable ones and they must disintegrate. Then the development of the standard theory of neutrino oscillations in the framework of particle physics is considered where the above mentioned shortcomings are absent and the oscillations of neutrino with equal masses are real ones and the oscillations of neutrino different masses are virtual ones. Expressions for probabilities of neutrino transitions (oscillations) in the correct theory are given.
\end{abstract}

PACS numbers: 14.60.Pq; 14.60.Lm

\section{Introduction}

The suggestion that, in analogy with $K^{o}, \bar{K}^{o}$ oscillations, there could be neutrino-antineutrino oscillations $(\nu \rightarrow \bar{\nu}$ ) was considered by Pontecorvo [1] in 1957. It was subsequently considered by Maki et al. [2] and Pontecorvo [3] that there could be mixings (and oscillations) of neutrinos of different flavors (i.e., $\nu_{e} \rightarrow \nu_{\mu}$ transitions). In the Standard theory of neutrino oscillations [4] is supposed that physical observed neutrino states $\nu_{e}, \nu_{\mu}, \nu_{\tau}$ have no definite masses and that they are directly created as mixture of the $\nu_{1}, \nu_{2}, \nu_{3}$ neutrino states. Below we discuss the consequences of these 
suppositions and then the correct theory of neutrino oscillations is considered.

\section{Remarks to the Theory of Neutrino Oscillations. Corrected Theory of Neutrino Oscillations}

At first, shortcomings of the Standard theory of neutrino oscillations are considered and then we pass to consideration of the corrected theory of neutrino oscillations.

\subsection{Remarks to the Standard Theory of Neutrino Oscillations}

In the Standard theory of neutrino oscillations [4], constructed in the framework of Quantum theory (Mechanics) in analogy with the theory of $K^{o}, \bar{K}^{o}$ oscillation, it is supposed that mass eigenstates are $\nu_{1}, \nu_{2}, \nu_{3}$ neutrino states but not physical observed neutrino states $\nu_{e}, \nu_{\mu}, \nu_{\tau}$. And that the neutrinos $\nu_{e}, \nu_{\mu}, \nu_{\tau}$ are directly created as superpositions of $\nu_{1}, \nu_{2}, \nu_{3}$ states (neutrinos). Then it is supposed that the $\nu_{e}, \nu_{\mu}, \nu_{\tau}$ neutrinos have no definite mass, i.e. their masses may vary in dependence on the $\nu_{1}, \nu_{2}, \nu_{3}$ admixture in the $\nu_{e}, \nu_{\mu}, \nu_{\tau}$ states. And also that neutrino oscillations are real oscillations even when their masses are different, i.e. that there is a real transition of electron neutrino $\nu_{e}$ into muon neutrino $\nu_{\mu}$ (or tau neutrino $\nu_{\tau}$ ). Obviously it is necessary to check up these suppositions. To simplify this, the case of two neutrinos is considered.

The mass lagrangian of two neutrinos $\left(\nu_{e}, \nu_{\mu}\right)$ has the following form $\left(\nu \equiv \nu_{L}\right):$

$$
\begin{gathered}
\mathcal{L}_{M}=-\frac{1}{2}\left[m_{\nu_{e}} \bar{\nu}_{e} \nu_{e}+m_{\nu_{\mu}} \bar{\nu}_{\mu} \nu_{\mu}+m_{\nu_{e} \nu_{\mu}}\left(\bar{\nu}_{e} \nu_{\mu}+\bar{\nu}_{\mu} \nu_{e}\right)\right] \equiv \\
\equiv-\frac{1}{2}\left(\bar{\nu}_{e}, \bar{\nu}_{\mu}\right)\left(\begin{array}{cc}
m_{\nu_{e}} & m_{\nu_{e} \nu_{\mu}} \\
m_{\nu_{\mu} \nu_{e}} & m_{\nu_{\mu}}
\end{array}\right)\left(\begin{array}{c}
\nu_{e} \\
\nu_{\mu}
\end{array}\right)
\end{gathered},
$$

which is diagonalized by rotation on the angle $\theta$ and then this lagrangian (1) transforms into the following one (see ref. in [4]):

$$
\mathcal{L}_{M}=-\frac{1}{2}\left[m_{1} \bar{\nu}_{1} \nu_{1}+m_{2} \bar{\nu}_{2} \nu_{2}\right]
$$


where

$$
m_{1,2}=\frac{1}{2}\left[\left(m_{\nu_{e}}+m_{\nu_{\mu}}\right) \pm\left(\left(m_{\nu_{e}}-m_{\nu_{\mu}}\right)^{2}+4 m_{\nu_{\mu} \nu_{e}}^{2}\right)^{1 / 2}\right]
$$

and angle $\theta$ is determined by the following expression:

$$
\begin{gathered}
\operatorname{tg} 2 \theta=\frac{2 m_{\nu_{e} \nu_{\mu}}}{\left(m_{\nu_{\mu}}-m_{\nu_{e}}\right)}, \\
\nu_{e}=\cos \theta \nu_{1}+\sin \theta \nu_{2}, \\
\nu_{\mu}=-\sin \theta \nu_{1}+\cos \theta \nu_{2} .
\end{gathered}
$$

Then $\nu_{e}, \nu_{\mu}$ masses are:

$$
\begin{aligned}
& m_{\nu_{e}}=m_{1} \cos ^{2} \theta+m_{2} \sin ^{2} \theta, \\
& m_{\nu_{\mu}}=m_{1} \sin ^{2} \theta+m_{2} \cos ^{2} \theta,
\end{aligned}
$$

i.e., $\nu_{e}, \nu_{\mu}$ neutrinos have definite masses which are expressed via $\nu_{1}, \nu_{2}$ masses and mixing angle $\theta$. It means that supposition that $\nu_{e}, \nu_{\mu}$ neutrinos have no definite masses is not confirmed.

If neutrino oscillations are real oscillations, i.e. there is a real transition of electron neutrino $\nu_{e}$ into muon neutrino $\nu_{\mu}$ (or tau- $\nu_{\tau}$ neutrino). Then the neutrino $x=\mu, \tau$ will decay in electron neutrino plus something

$$
\nu_{x} \rightarrow \nu_{e}+\ldots
$$

as a result, we get energy from vacuum, which is equal to the mass difference (if $m_{\nu_{x}}>m_{\nu_{e}}$ )

$$
\Delta E \sim m_{\nu_{x}}-m_{\nu_{e}}
$$

Then, again this electron neutrino transits into the muon neutrino, which decays again and we get energy and etc. So we have got a perpetuum mobile! Obviously, the law of energy and momentum conservation in these processes is not fulfilled.

Besides, since $\nu_{e}, \nu_{\mu}, \nu_{\tau}$ neutrinos are superpositions of $\nu_{1}, \nu_{2}, \nu_{3}$ then the $\nu_{e}, \nu_{\mu}, \nu_{\tau}$ neutrinos are wave packets having widths which are equal to mass differences of the composing components, i.e., $\nu_{1}, \nu_{2}, \nu_{3}$ neutrinos, $\Delta m \rightarrow m_{\nu_{2}}-m_{\nu_{1}}$ or $m_{\nu_{3}}-m_{\nu_{1}}$. Then these $\nu_{e}, \nu_{\mu}, \nu_{\tau}$ states (neutrinos) are 
unstable ones and must disintegrate for the time $t$ which is determinated by the uncertainty relation [5], i.e.,

$$
t \cong \frac{1}{\Delta m}
$$

It also means that the Sun neutrinos cannot reach the Earth as $\nu_{e}, \nu_{\mu}, \nu_{\tau}$ neutrino states. But in experiments [6,7] we see namely $\nu_{e}, \nu_{\mu}, \nu_{\tau}$ states (neutrinos) but not other states. Without any doubt this Standard theory requires a correction in order to get rid of the above mentioned defects.

\subsection{Corrected Theory of Neutrino Oscillations}

In the framework of the Quantum Mechanics [5] all the states are wave packets having widths and these states are unstable ones and they must disintegrate. In contrast to the Quantum Mechanics in the framework of the Particle Physics theory [8] all particles are stable ones or if they have widths then they must decay in the states (particles) with small masses.

The only way to restore the law of energy conservation is to demand that this process is virtual one if neutrinos have different masses. Then, these oscillations will be virtual ones and they are described in the framework of the uncertainty relations.

So, the correct theory of neutrino oscillations can be constructed only into the framework of the Particle Physics theory, where the conception of mass shell is present $[8,9]$. Besides, every particle must be created on its mass shell and it will be left on its mass shell while passing through vacuum.

In the considered theory of neutrino oscillations [9], constructed in the framework of the particle physics theory, it is supposed (following to the experiment) that:

1) The physical observable neutrino states $\nu_{e}, \nu_{\mu}, \nu_{\tau}$ are eigenstates of the weak interaction with $W, Z^{o}$ exchanges. And, naturally, the mass matrix of $\nu_{e}, \nu_{\mu}, \nu_{\tau}$ neutrinos is diagonal, i.e., the mass matrix of $\nu_{e}, \nu_{\mu}$ and $\nu_{\mu}$ neutrinos has the following diagonal form (since these neutrinos are created in the weak interactions it means that they are eigenstates of 
these interactions and their mass matrix must be diagonal):

$$
\left(\begin{array}{ccc}
m_{\nu_{e}} & 0 & 0 \\
0 & m_{\nu_{\mu}} & 0 \\
0 & 0 & m_{\nu_{\mu}}
\end{array}\right)
$$

Besides, all the available, experimental results indicate that the lepton numbers $l_{e}, l_{\mu}, l_{\tau}$ are well conserved, i.e. the standard weak interactions (with $W, Z^{o}$ bosons) do not violate the lepton numbers.

2) Then, to violate the lepton numbers, it is necessary to introduce an interaction violating these numbers. It is equivalent to introducing of the nondiagonal mass terms in the mass matrix of $\nu_{e}, \nu_{\mu}, \nu_{\tau}$ neutrinos:

$$
M\left(\nu_{e}, \nu_{\mu}, \nu_{\tau}\right)=\left(\begin{array}{ccc}
m_{\nu_{e}} & m_{\nu_{e} \nu_{\mu}} & m_{\nu_{e} \nu_{\tau}} \\
m_{\nu_{\mu} \nu_{e}} & m_{\nu_{\mu}} & m_{\nu_{\mu} \nu_{\tau}} \\
m_{\nu_{\tau} \nu_{e}} & m_{\nu_{\tau} \nu_{\mu}} & m_{\nu_{\mu}}
\end{array}\right)
$$

Diagonalizing this matrix [4]

$$
M\left(\nu_{e}, \nu_{\mu}, \nu_{\tau}\right)=V^{-1} M\left(\nu_{1}, \nu_{2}, \nu_{2}\right) V,
$$

we go to the $\nu_{1}, \nu_{2}, \nu_{3}$ neutrino mass matrix

$$
\left(\begin{array}{ccc}
m_{\nu_{1}} & 0 & 0 \\
0 & m_{\nu_{2}} & 0 \\
0 & 0 & m_{\nu_{3}}
\end{array}\right)
$$

where $V$ is neutrino mixings matrix $V$. Then the vector state $\Psi\left(\nu_{e}, \nu_{\mu}, \nu_{\tau}\right)$, of $\nu_{e}, \nu_{\mu}, \nu_{\tau}$ neutrinos

$$
\Psi\left(\nu_{e}, \nu_{\mu}, \nu_{\tau}\right)=\left(\begin{array}{c}
\nu_{e} \\
\nu_{\mu} \\
\nu_{\tau}
\end{array}\right)
$$

is transformed in the vector state $\Psi\left(\nu_{1}, \nu_{2}, \nu_{2}\right)$ of $\nu_{1}, \nu_{2}, \nu_{2}$ neutrinos

$$
\Psi\left(\nu_{e}, \nu_{\mu}, \nu_{\tau}\right)=V \Psi\left(\nu_{1}, \nu_{2}, \nu_{2}\right) .
$$

In the parameterization proposed by Maiani [10] $V$ has the following form:

$$
V=\left(\begin{array}{ccc}
1 & 0 & 0 \\
0 & c_{\gamma} & s_{\gamma} \\
0 & -s_{\gamma} & c_{\gamma}
\end{array}\right)\left(\begin{array}{ccc}
c_{\beta} & 0 & s_{\beta} \exp (-i \delta) \\
0 & 1 & 0 \\
-s_{\beta} \exp (i \delta) & 0 & c_{\beta}
\end{array}\right)\left(\begin{array}{ccc}
c_{\theta} & s_{\theta} & 0 \\
-s_{\theta} & c_{\theta} & 0 \\
0 & 0 & 1
\end{array}\right),
$$


where $\theta, \beta, \gamma$ and $\delta$ are angles of neutrino mixings and parameter of $C P$ violation.

Exactly like the case of $K^{o}$ mesons created in strong interactions, when mainly $K^{o}, \bar{K}^{o}$ mesons are produced but not $K_{1}, K_{2}$ mesons. In the considered case $\nu_{e}, \nu_{\mu}, \nu_{\tau}$, but not $\nu_{1}, \nu_{2}, \nu_{3}$, neutrino states are mainly created in the weak interactions (this is so since contribution of the lepton numbers violating interactions in this process is too small and in this case no oscillations take place).

3) Then, when the $\nu_{e}, \nu_{\mu}, \nu_{\tau}$ neutrinos are passing through vacuum, they will be converted into superpositions of the $\nu_{1}, \nu_{2}, \nu_{3}$ owing to the presence of the interactions violating the lepton number of neutrinos and will be left on their mass shells. And, then, oscillations of the $\nu_{e}, \nu_{\mu}, \nu_{\tau}$ neutrinos will take place according to the standard scheme [4]. Whether these oscillations are real or virtual, it will be determined by the masses of the physical observed neutrinos $\nu_{e}, \nu_{\mu}, \nu_{\tau}$.

i) If the masses of the $\nu_{e}, \nu_{\mu}, \nu_{\tau}$ neutrinos are equal, then the real oscillation of the neutrinos will take place.

ii) If the masses of the $\nu_{e}, \nu_{\mu}, \nu_{\tau}$ are not equal, then the virtual oscillation of the neutrinos will take place. To make these oscillations real, these neutrinos must participate in the quasielastic interactions, in order to undergo transition to the mass shell of the other appropriate neutrinos in analogy with $\gamma-\rho^{o}$ transition in the vector meson dominance model. It is necessary to take into account that in contrast to the strong interactions, the dependence on squared transferring momentum in the weak interactions has flat form since $W$ boson has a huge mass. In case ii) enhancement of neutrino oscillations will take place if the mixing angle is small at neutrinos passing through a bulk of matter [11].

So the neutrino mixings (oscillations) appear due to the fact that at neutrino creating the eigenstates of the weak interactions (i.e. $\nu_{e}, \nu_{\mu}, \nu_{\tau}$ neutrino states) are produced but not the eigenstates of the weak interaction violating lepton numbers (i.e. $\nu_{1}, \nu_{2}, \nu_{3}$ neutrino states). And then when neutrinos are passing through vacuum they are converted into superpositions of $\nu_{1}, \nu_{2}, \nu_{3}$ neutrinos. If $\nu_{1}, \nu_{2}, \nu_{3}$ neutrinos were originally created, then the mixings (oscillations) would not have taken place since the weak interaction conserves the lepton numbers. 
In the case of three neutrino types the probability of $\nu_{e} \rightarrow \nu_{e}$ transitions has the following form:

$$
\begin{gathered}
P\left(\nu_{e} \rightarrow \nu_{e}, t\right)=1-\cos ^{4}(\beta) \sin ^{2}(2 \theta) \sin ^{2}\left(-t\left(E_{1}-E_{2}\right) / 2\right)- \\
\cos ^{2}(\theta) \sin ^{2}(2 \beta) \sin ^{2}\left(-t\left(E_{1}-E_{3}\right) / 2\right)- \\
-\sin ^{2}(\theta) \sin ^{2}(2 \beta) \sin ^{2}\left(-t\left(E_{2}-E_{3}\right) / 2\right)
\end{gathered}
$$

where $E_{1}, E_{2}, E_{3}$ are energy of $\nu_{1}, \nu_{2}, \nu_{3} \rightarrow x$ neutrinos and $E_{x}=\sqrt{p^{2}+m_{x}^{2}}$.

Since lengths of neutrino oscillations

$$
L_{i, j}=2 \pi \frac{2 p}{\left|m_{2}^{2}-m_{1}^{2}\right|} \quad i \neq j=1,2,3 .
$$

are different, then the expression of probability for neutrino oscillations at small distances has a simpler form. For example, for $\nu_{e} \rightarrow \nu_{e}$ oscillations we have

$$
P\left(\nu_{e} \rightarrow \nu_{e}\right)=1-\sin ^{2} 2 \theta \sin ^{2}\left(\left(m_{2}^{2}-m_{1}^{2}\right) / 2 p\right) t
$$

where

$$
\sin ^{2} \theta=1 / 2-\frac{\left(m_{\nu_{e}}-m_{\nu_{\mu}}\right)}{2 \sqrt{\left(m_{\nu_{e}}-m_{\nu_{\mu}}\right)^{2}+\left(2 m_{\nu_{e} \nu_{\mu}}\right)^{2}}},
$$

and

$$
\sin ^{2} 2 \theta=\frac{\left(2 m_{\nu_{e} \nu_{\mu}}\right)^{2}}{\left(m_{\nu_{e}}-m_{\nu_{\mu}}\right)^{2}+\left(2 m_{\nu_{e} \nu_{\mu}}\right)^{2}},
$$

It is interesting to remark that expression (20) can be obtained from the Breit-Wigner distribution [12]

$$
P \sim \frac{(\Gamma / 2)^{2}}{\left(E-E_{0}\right)^{2}+(\Gamma / 2)^{2}}
$$

by using the following substitutions:

$$
E=m_{\nu_{e}}, \quad E_{0}=m_{\nu_{\mu}}, \Gamma / 2=2 m_{\nu_{e}, \nu_{\mu}},
$$

where $\Gamma / 2 \equiv W(\ldots)$ is a width of $\nu_{e} \leftrightarrow \nu_{\mu}$ transitions, i.e., virtual neutrino oscillations keeps in within the uncertainty relation. In the general case these widths can be computed by using a standard method [13]. 
If $m_{\nu_{e}, \nu_{\mu}}$ differs from zero, then Exp. (20) gives a probability of $\nu_{e} \leftrightarrow \nu_{\mu}$ transitions and then the probability of $\nu_{e} \leftrightarrow \nu_{\mu}$ transitions is defined by these neutrino masses and width of their transitions. If $m_{\nu_{e}, \nu_{\mu}}=0$, then the $\nu_{e} \leftrightarrow \nu_{\mu}$ transitions are forbidden. So, this is a solution of the problem of the origin of the mixing angle in the theory of vacuum oscillations.

It is necessary to remark that in this correct theory of neutrino oscillations, in contrast to the Standard theory, oscillations of neutrinos with equal masses are real ones and the oscillations of neutrinos with different masses are virtual ones and then the problem of energy momentum conservation and the problem of neutrino disintegrations as wave packets are solved.

In the above considered theory of neutrino oscillations neutrino masses change at neutrino oscillations (for example $m_{\nu_{e}} \rightarrow m_{\nu_{\mu}}$ ). Theoretically it is also possible neutrino transitions without changing their masses [13]. In this case the mixing angles are maximal $(\pi / 4)$. The author proposed another mechanism (model) of neutrino transitions which is analogous to the model of vector dominance, i.e., the model of $\gamma \rightarrow \rho^{o}$ transitions [14].

\section{Conclusions}

In the Standard theory of neutrino oscillations it is supposed that physical observed neutrino states $\nu_{e}, \nu_{\mu}, \nu_{\tau}$ have no definite masses and that they are initially created as mixture of the $\nu_{1}, \nu_{2}, \nu_{3}$ neutrino states and that neutrino oscillations are real ones even when their masses are different. It was shown that these suppositions lead to violation of the law of energy and momentum conservation and then the neutrino states are unstable ones and they must disintegrate. Then the development of the Standard theory of neutrino oscillations in the framework of particle physics has been considered where the above mentioned shortcomings are absent and the oscillations of neutrino with equal masses are real ones and the oscillations of neutrino different masses are virtual ones. Expressions for probabilities of neutrino transitions (oscillations) in the corrected theory have been given. 


\section{References}

1. Pontecorvo B. M., Soviet Journ. JETP, 1957, v. 33, p.549;

JETP, 1958, v.34, p.247.

2. Maki Z. et al., Prog.Theor. Phys., 1962, vol.28, p.870.

3. Pontecorvo B. M., Soviet Journ. JETP, 1967, v. 53, p.1717.

4. Bilenky S.M., Pontecorvo B.M., Phys. Rep., C41(1978)225;

Boehm F., Vogel P., Physics of Massive Neutrinos: Cambridge

Univ. Press, 1987, p.27, p.121;

Bilenky S.M., Petcov S.T., Rev. of Mod. Phys., 1977, v.59, p.631.

Gribov V., Pontecorvo B.M., Phys. Lett. B, 1969, vol.28, p.493.

5. Schiff L. I., Quantum Mechanics, McRam, ..., London, 1955.

Kayser B., Phys. Rev. D24, 1981, p.110.

6. Kameda J., Proceedings of ICRC 2001, August 2001, Germany,

Hamburg, p.1057.

Fukuda S. et al,. Phys. Rev. Lett., 2001, v.25, p.5651;

Phys. Lett. B, 539, 2002, p.179.

7. Ahmad Q. R. et al., Internet Pub. nucl-ex/0106015, June 2001.

Ahmad Q. R. et al., Phys. Rev. Lett. 2002, v. 89, p.011301-1;

Phys. Rev. Lett. 2002,v. 89, p.011302-1.

8. Schweber S., An Introduction to Relativistic Quantum Field Theory,

Row, ..., New York, 1961.

Ta-Pei Cheng, Ling-Fong Li, Gauge Theory of Elementary Particle

Physics, Clarendon Press-Oxford, 1984.

9. Beshtoev Kh.M., JINR Commun. E2-92-318, Dubna, 1992;

JINR Rapid Communications, N3[71]-95.

hep-ph/9911513;

The Hadronic Journal, v.23, 2000, p.477;

Proceedings of 27th Intern. Cosmic Ray Conf., Germany,

Hamburg, 7-15 August 2001, v.3, p. 1186.

10. L. Maiani, Proc. Int. Symp. on Lepton-Photon

Inter., Hamburg, DESY, p.867.

11. Beshtoev Kh.M., JINR Commun, E2-93-297, Dubna, 1993;

JINR Commun. E2-94-46; Hadronic Journal, 1995, vol 18, p.165. 
12. Blatt J.M., Weiscopff V.F., The Theory of Nuclear Reactions, INR T.R. 42.

13. Beshtoev Kh.M., hep-ph/9911513;

The Hadronic Journal, v.23, 2000, p.477;

Proceedings of 27th Intern. Cosmic Ray Conf., Germany, Hamburg, 7-15 August 2001, v.3, p. 1186.

Beshtoev Kh.M., JINR Commun. E2-99-307, Dubna, 1999;

JINR Commun. E2-99-306, Dubna, 1999.

14. Sakurai J.J., Currents and Mesons, The Univ of Chicago Press, 1967.

Beshtoev Kh. M., JNR of USSR Academy Science P-217,

Moscow, 1981. 